\title{
Input Selection Based on an Ensemble
}

\author{
Piërre van de Laar and Tom Heskes \\ RWCP* Novel Functions SNN $^{\dagger}$ Laboratory \\ Department of Medical Physics and Biophysics, \\ University of Nijmegen , \\ Nijmegen, The Netherlands \\ email: \{ pierre, tom \}@mbfys.kun.nl
}

\begin{abstract}
Since an ensemble of neural networks outperforms a single network, we expect that the selection of input variables based on an ensemble is superior to the selection based on a single neural network. In this article, we will present an algorithm that performs input selection based on an ensemble of neural networks. Using this algorithm, the correct sets of variables were found for two artificial problems. Furthermore, for two real-world problems, we determined the relevance of the input variables. Our predictions were equal or better than the predictions of other methods described in the literature.
\end{abstract}

\section{Keywords}

architecture selection, combining classifiers, combining predictors, feature selection, input selection, knowledge extraction, subset selection.

\section{Introduction}

Whenever a neural network is trained on only part of the available data, the resulting solution will strongly depend on the actually selected data examples (Breiman 1996). This "instability" effect is particularly strong when the number of examples is rather small, as is the case in many real-world problems. An ensemble of neural networks, trained on different parts of the available data, will therefore contain different, maybe even contradicting, solutions. The existence of these different solutions does not only reflect the uncertainty caused by the limited amount of data, but also explains why an ensemble outperforms a single neural network (Breiman 1996, Heskes 1997). Therefore, input selection should prefer an ensemble to a single network. Although many input selection algorithms have been proposed for single neural networks, see, for example, (Belue \& Bauer 1995, Glorfeld 1996, Grandvalet \& Canu 1997, Refenes et al. 1996, van de Laar et al. 1997), we are not aware of any input selection algorithm based on an ensemble of neural networks. Unfortunately, approaches to select input variables from single neural networks cannot easily be generalised to an ensemble, since one should not apply schemes that treat the ensemble as

\footnotetext{
* Real World Computing Partnership

$\dagger^{\dagger}$ Foundation for Neural Networks
} 
a whole, such as for example majority voting, and ignore the conflicts within the ensemble, the cause of the improvement in performance.

Before proposing an algorithm for input selection based on ensembles, in Section 4, we will first describe how we create an ensemble of neural networks (see Section 2) and give a brief introduction to input selection (see Section 3). Simulations of this algorithm and their results are described in Section 5. We will end with our conclusions and discussion (see Section 6).

\section{Ensemble}

We will now describe how we create an ensemble of neural networks. For each neural network in the ensemble the (training) data is randomly divided into estimation and validation data. Starting from small random initial values, the weights of the network are updated through backpropagation of the mean squared error on the estimation data. Training is stopped at the minimum of the mean squared error on the validation data.

In this article we will also use some real-world datasets. For the sole purpose of comparison, the available data is first divided into test and training data. Using the training data an ensemble of neural networks is created exactly as is described before. The test data, which is never used during training, is used to estimate the performance of this ensemble and to compare the performances of different input selection algorithms.

\section{Input selection}

If we want to determine which of the $N$ variables should be included for a given task, we have to consider $2^{N}$ possible sets of variables. Therefore, a procedure that estimates the relevance of the variables based on the performance of every possible set of input variables, is only feasible if the number of input variables is rather small. Consequently, instead of an exhaustive search, in real world application we often use approximations, such as backward elimination, forward selection, and stepwise selection [see e.g. (Draper \& Smith 1981, Kleinbaum et al. 1988)].

To estimate the performance of a single neural network on a given set of variables, we used an algorithm called "partial retraining". We chose this algorithm since it outperformed all other algorithms such as Optimal Brain Surgeon, weight analysis, and constant substitution in a benchmark study (van de Laar et al. 1997).

\section{Algorithm}

When one performs input selection on the ensemble as a whole, for example, by selecting the input variables according to the majority of the individual neural networks in the ensemble, one ignores the conflicting solutions within the ensemble that are the source of the improvement in generalising performance (Breiman 1996, Heskes 1997). On the other hand, when one performs input selection on each of the neural networks of the ensemble separately, one might not be able to combine these individual selections into a single comprehensible input selection scheme. We propose an algorithm that lies between these two extremes and that is closely related to backward elimination. 
In the remaining part of this section we will first introduce our notation, then we will describe the algorithm and illustrate it on an example. We will end with a brief discussion of the computational cost of the algorithm.

\subsection{Notation}

The (estimated) error on the task of a single neural network $i$ given the set $s$ of input variables will be denoted by $E_{s}^{i}$. The minimal error of neural network $i$ given a collection of sets $V$ is denoted by $E_{\min }^{i}(V)=\min _{s \in V} E_{s}^{i}$. For example, if $V$ contains two sets $\bar{s}=\{1,4,7,9\}$ and $s^{*}=\{3,5,8\}$, i.e., $V=$ $\{\{1,4,7,9\},\{3,5,8\}\}$, then $E_{\min }^{i}(V)$ is the minimal error of network $i$ on either $\bar{s}$ or $s^{*}$. And finally, the minimal error of the ensemble given a collection of sets $V$ is denoted by $M(V)=\sum_{i} E_{\min }^{i}(V)$, where the sum is calculated over all neural networks in the ensemble. Note that different neural networks in the ensemble might attain their minimal error on different sets.

\subsection{Description}

Starting with a collection of sets $V$ that only contains one set containing all variables, the algorithm repeats the following steps until all variables are removed.

1. Based on the collection of sets $V$ determine all possible sets with one variable less.

2. For each neural network in the ensemble estimate its generalising performance on these sets. In the simulations the generalising performance will be estimated by partial retraining the neural network based on the estimation data and calculating its performance on the validation data.

3. Determine the set $s^{*}$ that yields the lowest error, i.e., $s^{*}=\underset{s}{\operatorname{argmin}} \sum_{i} E_{s}^{i}$, where the sum is calculated over all neural networks in the ensemble.

4. Redefine the collection of sets $V$ such that it only contains the set $s^{*}$. The corresponding minimal error is thus equal to $M(V)$.

5. From the remaining sets, find the set $\bar{s}$ that yields the largest decrease in minimal error when this set would be included in the collection of sets $V$. Of course, this set also gives the lowest minimal error when included, i.e., $\bar{s}=\underset{s \notin V}{\operatorname{argmin}} M(\{V, s\})$.

6. If the largest relative decrease in error, i.e., the largest decrease in the error divided by the minimal error, is larger than the parameter $\gamma$, add the corresponding set $\bar{s}$ to the collection of sets $V$ and go back to the previous step.

Our algorithm only depends on the parameter $\gamma^{1}$. This parameter controls the comprehensibility of the input selection scheme versus the accuracy of this scheme. If a small value is chosen for this parameter, it is very likely that many sets with slightly different solutions will be allowed within the collection of sets. If a large value is chosen, probably only a few sets with very different solutions will exist within the collection of sets. Following these steps, it can be easily

\footnotetext{
${ }^{1}$ In our simulation we observed good results with $\gamma \in\left[10^{-3}, 10^{-1}\right]$.
} 
Table 1: Estimated performance of each network on each possible set (first sweep).

\begin{tabular}{r|r|r|r} 
Set & $E_{s}^{1}$ & $E_{s}^{2}$ & $\sum_{i} E_{\min }^{i}(s)$ \\
\hline$\{1,2,3\}$ & 48 & 50 & 98 \\
$\{1,2,4\}$ & 48 & 46 & 94 \\
$\{1,3,4\}$ & 46 & 50 & 96 \\
$\{2,3,4\}$ & 44 & 48 & 92
\end{tabular}

Table 2: Estimated performance of each network on a number of collections of sets (first sweep).

\begin{tabular}{r|r|r|r} 
Collection of sets & $E_{\min }^{1}(V)$ & $E_{\min }^{2}(V)$ & $\sum_{i} E_{\min }^{i}(V)$ \\
\hline$\{\{1,2,3\}\}$ & 48 & 50 & 98 \\
$\{\{1,2,4\}\}$ & 48 & 46 & 94 \\
$\{\{1,3,4\}\}$ & 46 & 50 & 96 \\
$\{\{2,3,4\}\}$ & 44 & 48 & 92 \\
\hline$\{\{1,2,3\},\{2,3,4\}\}$ & 44 & 48 & 92 \\
$\{\{1,2,4\},\{2,3,4\}\}$ & 44 & 46 & 90 \\
$\{\{1,3,4\},\{2,3,4\}\}$ & 44 & 48 & 92
\end{tabular}

seen that our algorithm only considers collections of sets with the same number of input variables. Furthermore, our algorithm not only iteratively finds the sets of input variables but also clusters the different solutions within the ensemble of neural networks.

\subsection{Example}

To illustrate our algorithm we will now give a rather lengthy example. We assume that we have an ensemble of two neural networks and a database that contains four input variables. The parameter $\gamma$ is set to $2.5 \%$.

\subsubsection{First sweep}

The algorithm starts with the collection of one set that contains all variables, i.e., $\{\{1,2,3,4\}\}$. In the first step all possible sets with one variable less are determined. In this case this will result in $\{\{1,2,3\},\{1,2,4\},\{1,3,4\},\{2,3,4\}\}$. In step two, for each network the generalising performance on these sets are estimated and are given in Table 1 . In step three, after calculating the errors of the sets $\{1,2,3\},\{1,2,4\},\{1,3,4\}$ and $\{2,3,4\}$, which are equal to 98,94 , 96 and 92 , respectively, the set $\{2,3,4\}$ that yields the lowest error (92) is determined. In step four we redefine $V$ to be $\{\{2,3,4\}\}$, i.e., it contains only the set with the lowest error. Consequently, $M(V)$ is equal to 92 . By removing the set $\{2,3,4\}$ from all possible sets with one variable less, we reduce the number of elements in this collection, and end up with $\{\{1,2,3\},\{1,2,4\},\{1,3,4\}\}$. In step five, we determine the decrease in error when one of these sets would be added to our collection of sets $V$. Again using Table 1, we can easily determine the error on these collections of sets. As can be seen in Table 2, adding the set $\{1,2,4\}$ to $V$, resulting in the collections of sets $\{\{1,2,4\},\{2,3,4\}\}$, will yield the largest decrease in error (of size 2). Since the relative decrease in error $(2 / 92)$ is less than $2.5 \%$ we will not add the set $\{1,2,4\}$ to the collection of sets 
Table 3: Estimated performance of each network on a number of collections of sets (second sweep).

\begin{tabular}{r|r|r|r} 
Collection of sets & $E_{\min }^{1}(V)$ & $E_{\min }^{2}(V)$ & $\sum_{i} E_{\min }^{i}(V)$ \\
\hline$\{\{2,3\}\}$ & 48 & 52 & 100 \\
$\{\{2,4\}\}$ & 52 & 46 & 98 \\
$\{\{3,4\}\}$ & 46 & 50 & 96 \\
\hline$\{\{2,3\},\{3,4\}\}$ & 46 & 50 & 96 \\
$\{\{2,4\},\{3,4\}\}$ & 46 & 46 & 92 \\
\hline$\{\{2,3\},\{2,4\},\{3,4\}\}$ & 46 & 46 & 92
\end{tabular}

Table 4: Estimated performance of each network on a number of collections of sets (third sweep).

\begin{tabular}{r|r|r|r} 
Collection of sets & $E_{\min }^{1}(V)$ & $E_{\min }^{2}(V)$ & $\sum_{i} E_{\min }^{i}$ \\
\hline$\{\{2\}\}$ & 56 & 54 & 110 \\
$\{\{3\}\}$ & 58 & 56 & 114 \\
$\{\{4\}\}$ & 50 & 54 & 104 \\
\hline$\{\{2\},\{4\}\}$ & 50 & 54 & 104 \\
$\{\{3\},\{4\}\}$ & 50 & 54 & 104
\end{tabular}

$V$. Therefore, we are finished and we return to the first step.

\subsubsection{Second sweep}

Since $V$ is now equal to $\{\{2,3,4\}\}$ step one results in $\{\{2,3\},\{2,4\},\{3,4\}\}$. In step two the performances are estimated. These performances can be found in Table 3. In step three we find that $s^{*}=\{3,4\}$. Thus in step four $V$ is made equal to $\{\{3,4\}\}$ and $M(V)$ is thus equal to 96 . In step five, we determine the decrease in error when one of the remaining sets would be added to our collection of sets $V$. Using Table 3 , we see that adding the set $\{2,4\}$ to $V$ results in an improvement of 4 , where as adding the set $\{2,3\}$ yields no improvement. So, since the relative decrease in error $(4 / 96)$ is more than $2.5 \%$ we add the set $\{2,4\}$ to our collection of sets $V$, set $M(V)$ equal to 92 and we go back to step five. We again determine the decrease in error when one of the remaining sets would be added to our current collection of sets $V=\{\{2,4\},\{3,4\}\}$. Again using Table 3 , we see that adding the (only remaining) set $\{2,3\}$ to $V$ yields no improvement. Therefore, in step six we conclude that the decrease in minimal error does not justify adding another set to our collection of sets $V$, and we again go back to step one.

\subsubsection{Third sweep}

Since $V$ is now equal to $\{\{2,4\},\{3,4\}\}$ step one results in $\{\{2\},\{3\},\{4\}\}$. In step two the performances are estimated and they can be found in Table 4 . In step three we find that $s^{*}=\{4\}$. Thus in step four $V$ is made equal to $\{\{4\}\}$ and $M(V)$ is equal to 104. In step five, we determine the decrease in error when one of the remaining sets would be added to our collection of sets $V$. Using Table 4, we see that neither adding the set $\{2\}$ or $\{3\}$ to $V$ results in any improvement. Therefore, in step six we conclude that the increase in performance does not justify adding a set to our collection of set $V$, and we go back to step one, for 


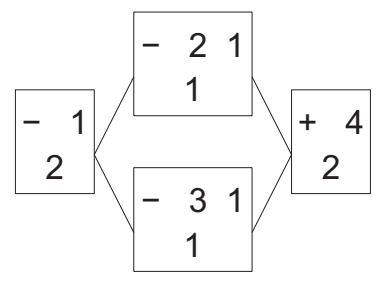

Figure 1: Summarising graph of our example. See text for explanation.

the last time.

\subsubsection{Final sweep}

Since $V$ is equal to $\{\{4\}\}$ step one now results in $\{\emptyset\}$. In step two we now determine the (base) performance. Since the empty set is the only set it will be selected in step three as the set with the lowest error. And thus in step four $V$ will contain the empty set, and $M(V)$ is equal to the base performance, i.e., the performance with no input variables. Step five and six are skipped since there are no remaining sets. And finally our algorithm ends since all variables have been removed.

\subsection{Graph}

Normally we will not give all these details of the algorithm but we will summarise the results in a single graph. The graph that summarises our example is given in Figure 1. Each box in this graph contains two lines. The number on the lower line denotes the number of neural networks whose validation error is minimal on this set from all displayed sets. The symbols on the upper line indicate the set of input variables. To indicate a set two notations are possible. First, one could indicate which elements are contained in the set. Second, one could indicate which elements have to be removed to arrive at this set. In our example, the set $\{2,4\}$ can be made by removing the elements one and three from the initial set containing all elements [ -13$]$ or by adding the elements two and four to the empty set $[+24]$. For brevity of notation and thus readability of the graph, we will always use the shortest notation. Therefore, in the beginning the elements to be removed will be indicated, but when more than half of all elements have been removed we will indicate the remaining variables. To distinguish between these two notations we will use a minus sign to denote removal and a plus sign to denote inclusion. As a final remark, when two or more sets of the same size contain (or remove) the same elements we will emphasize this by first giving the elements in which they differ and then giving the elements on which they agree. Therefore, in Figure 1 we have written [ - 21 ] and [ - 31 ] rather than [ - $\left.\begin{array}{ll}1 & 2\end{array}\right]$ and $\left[\begin{array}{lll}-1 & 3\end{array}\right]$.

\subsection{Computational Cost}

An ensemble of neural networks often outperforms a single network, yet this increase in performance is achieved at the expense of an increase in computational cost. Whenever an ensemble is used instead of a single network, the computational cost will at least increase first, by a factor equal to the size of the ensemble and second, due to additional overhead. Also our algorithm shows 


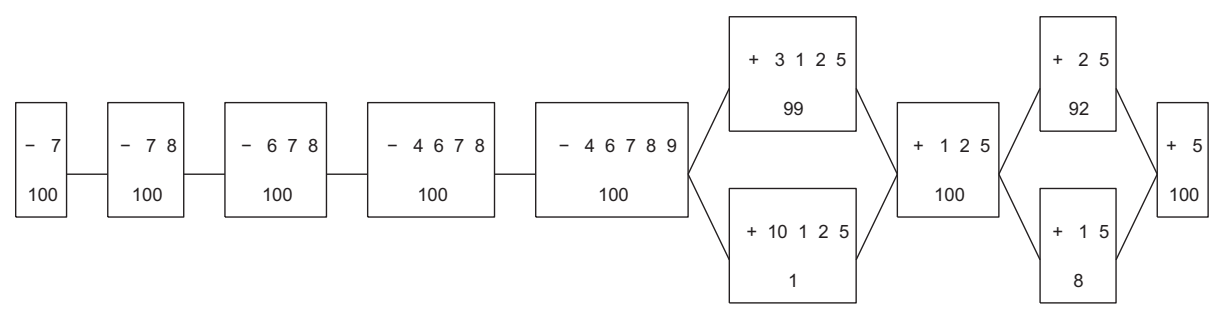

Figure 2: The sets of the input variables of the signal plus noise problem as determined by an ensemble of one hundred neural networks with $\gamma=0.05$. See Section 4.4 for explanation.

this kind of increase in computational cost when compared to input selection algorithms based on single networks. Furthermore, a comparison between our algorithm based on an ensemble containing a single network and more than one network shows that additional sets might be considered and thus additional computational costs have to be accepted. In our previous example, when the ensemble would contain only network one or two instead of both then 16 collections of sets would be considered whereas now 19 collections of sets have been considered. Although the number of different collections to be considered depends, amongst others, on the data set, we have some control over it by our parameter $\gamma$.

\section{Simulations}

We determined and visualised the sets of input variables for two artificial and two real-world tasks using an ensemble that contained one hundred two-layered perceptrons with the hyperbolic tangent and the identity as transfer functions of the hidden and output layer, respectively.

\subsection{Regression}

The response of our artificial regression task given ten input variables, i.e., $X_{1}, \ldots, X_{10}$, is given by the following signal plus noise model (Friedman 1991)

$$
T=10 \sin \left(\pi X_{1} X_{2}\right)+20\left(X_{3}-\frac{1}{2}\right)^{2}+10 X_{4}+5 X_{5}+\epsilon,
$$

where $\epsilon$ is $\mathcal{N}(0,1)$, i.e., standard normally distributed noise. Note that the response does not depend on the irrelevant input variables, $X_{6}, X_{7}, X_{8}, X_{9}$, and $X_{10}$. Each of the input variables is drawn from a uniform distribution over $[0,1]$, but the input variables are dependent: two irrelevant inputs are identical, $X_{9} \equiv X_{10}$, as well as two relevant inputs, $X_{4} \equiv X_{5}$.

All neural networks in the ensemble had ten inputs, seven hidden units, and one output, and were trained as previously described using 200 estimation and 200 validation patterns. See Figure 2 for the sets of input variables we derived with $\gamma$ equal to 0.05 . Figure 2 shows that all neural networks, except one (which is confused by the redundant irrelevant variable $X_{10}$ ), correctly learned the structure of the problem since the right sets of input variables were detected.

We have applied our algorithm on the Boston housing data (Belsley et al. 1980), a well-known real-world regression task. All neural networks in the ensemble had thirteen inputs, five hidden units, and one output, and were trained 


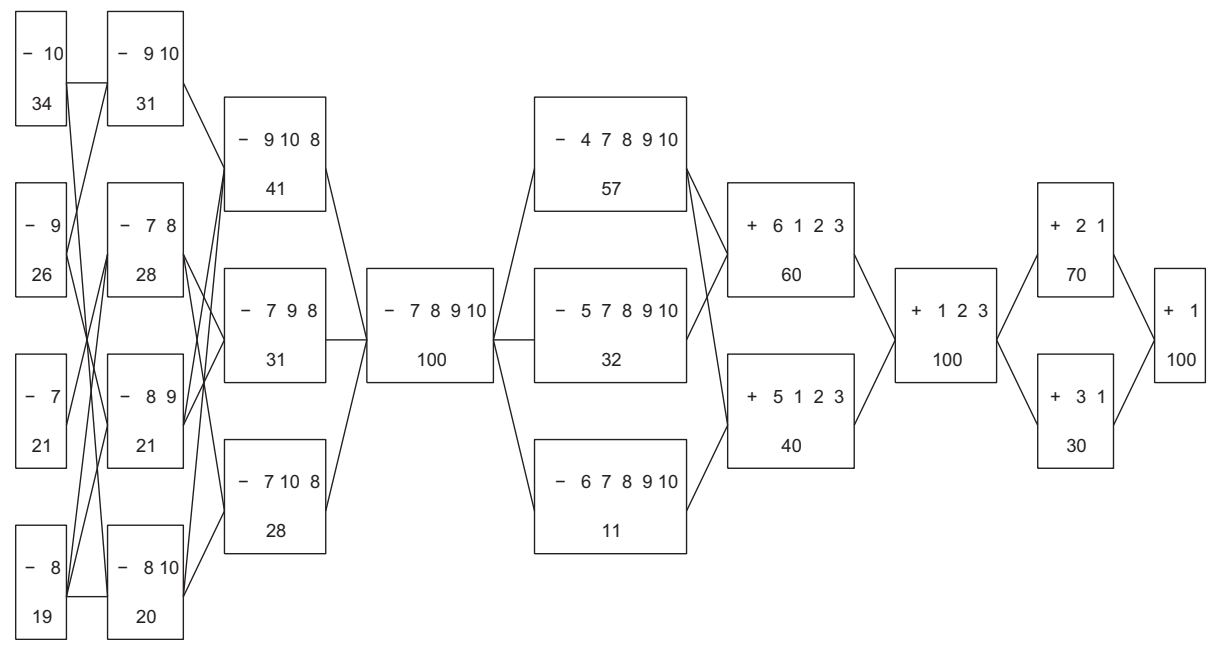

Figure 3: The sets of the input variables of the rule problem as found by an ensemble of a hundred neural networks with $\gamma=0.01$. See Section 4.4 for explanation.

using 253 estimation and 253 validation patterns. For $\gamma=0.05$ all hundred neural networks in the ensemble agreed that the variables are in order of decreasing importance: $13,6,9,11,8,1,5,4,2,7,10,12$, and 3 . In (Hofmann \& Tresp 1996) an exhaustive search in a Bayesian network is applied to determine the structure and relevant variables of the Boston housing data. The relevant variables according to (Hofmann \& Tresp 1996) are $X_{5}, X_{6}, X_{8}, X_{10}, X_{11}$, and $X_{13}$.

To compare the predictions of (Hofmann \& Tresp 1996) and our algorithm the 506 data patterns were randomly divided in 106 test and 400 training patterns. We trained three ensembles by randomly selecting 200 estimation and 200 validation patterns for each network. One ensemble was trained using all input variables. The input of the other two ensembles was restricted to the six most important variables according to the two algorithms. Averaged over fifty runs, the ensembles with all thirteen input variables had a worse performance $(10.8 \pm 0.5)$ than both the two ensembles with restricted input. The ensemble with input variables as given by (Hofmann \& Tresp 1996) was not significantly better than our ensemble $(9.1 \pm 0.5$ versus $9.3 \pm 0.5)$.

\subsection{Classification}

The response of our artificial classification task given the ten binary input variables, $X_{1}, \ldots, X_{10}$ is given by the following rule

$$
T=X_{1} \vee\left(\overline{X_{2}} \wedge X_{3}\right) \vee\left(X_{4} \wedge \overline{X_{5}} \wedge \overline{X_{6}}\right) \text {, }
$$

where 0 and 1 code false and true, respectively. The response thus depends on one very important variable $\left(X_{1}\right)$, two equally important variables $\left(X_{2}\right.$ and $\left.X_{3}\right)$, and three less important variables $\left(X_{4}, X_{5}\right.$, and $\left.X_{6}\right)$. Furthermore, the response does not depend on the four irrelevant or noisy variables, $X_{7}, X_{8}, X_{9}$, and $X_{10}$.

All neural networks in the ensemble had ten inputs, five hidden units, and one output, and were trained as described above using estimation and validation 


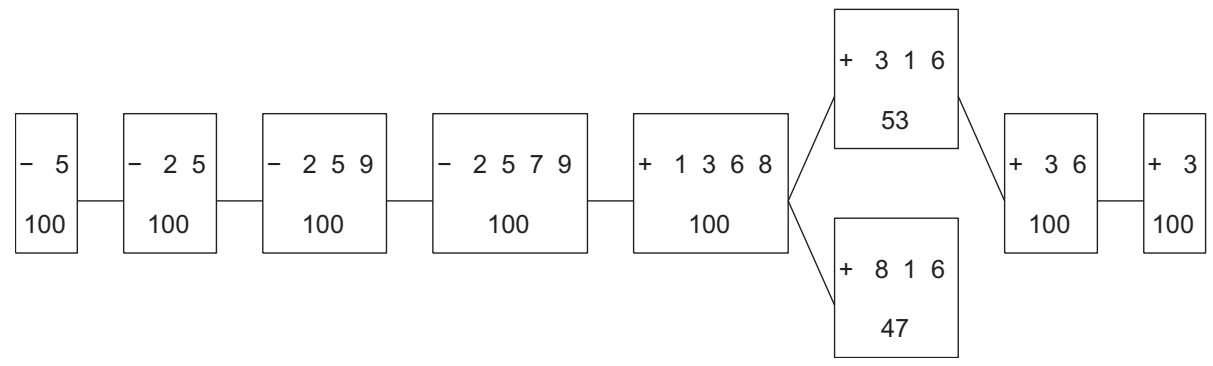

Figure 4: The sets of the input variables of the Wisconsin Breast Cancer Database as derived from an ensemble of a hundred neural networks with $\gamma=0.05$. See Section 4.4 for explanation.

data both containing 200 patterns. See Figure 3 for the sets of input variables as found by an ensemble of a hundred neural networks with $\gamma=0.01$.

In Figure 3, we see that the neural networks did not all learn the same solution to the problem, since they disagreed on the relevance of, for example, the theoretically equally important variables four, five, and six. Furthermore, we can see the structure of the rule problem, i.e., one very important, two equally important, three less important, and four irrelevant variables.

Finally, our algorithm was applied on the Wisconsin Breast Cancer Database (Wolberg \& Mangasarian 1990). In this real-world classification task one has to predict, based on nine input variables, whether or not a tumor is malignant. All neural networks in the ensemble had nine inputs, five hidden units, and one output, and were trained using 350 estimation and 349 validation patterns. See Figure 4 for the sets of input variables as found by an ensemble of a hundred neural networks with $\gamma$ equal to 0.05 .

Like (Setiono \& Liu 1997), we conclude that the two most relevant variables are attribute 3 and 6 . But we disagree on the next three ${ }^{2}$ most relevant variables. To compare our algorithm's prediction with those of (Setiono \& Liu 1997), the 699 data patterns were randomly divided in 199 test and 500 training patterns. We trained the networks in three ensembles as previously described using 250 estimation and 250 validation patterns. The input of the neural networks of the first ensemble was restricted to $X_{3}, X_{4}, X_{6}, X_{8}$, and $X_{9}$, the five most important variables according to (Setiono \& Liu 1997). The input of the second ensemble was restricted to the five most important variables according to our algorithm, i.e., $X_{1}, X_{3}, X_{4}, X_{6}$, and $X_{8}$. The last ensemble received all variables as its input. Averaged over fifty runs, the ensemble with its input restricted according to (Setiono \& Liu 1997) was worse $(7.0 \pm 0.3)$ than the other two ensembles. The ensemble with the input variables restricted according to our algorithm was slightly, but not significantly, better than the ensemble with all input variables $(6.3 \pm 0.3$ versus $6.4 \pm 0.3)$.

\footnotetext{
${ }^{2}$ If (Setiono \& Liu 1997) would have specified which three variables were considered the most relevant, the comparison of our methods would be even more interesting, since our algorithm does not define "the" third most important variable, but yields an ensemble in which two different solutions are included.
} 


\section{Discussion}

In this article, we described an algorithm for input selection based on an ensemble of neural networks. In two artificial problems, we showed that the correct sets were selected. For two real-world problems, we found solutions which were at least as good as other solutions given in the literature. Furthermore, although input selection based on a single neural network is an unstable process, i.e., a small change can yield a completely different selection, input selection based on an ensemble will be more stable due to the averaging over a large number of neural networks.

The algorithm which we proposed only stops when all variables have been removed. Afterwards, we can determine the optimal ensemble for this task with some additional statistical test, see for example (van de Laar et al. 1997). As already described, our algorithm only considers ensembles which are composed of neural networks with the same number of input variables. Since these specific ensembles constitute a subset of ensembles of neural networks, we prefer to consider our results only as a guideline. For example, our predictions whether a breast cancer tumor is malignant might be based on an ensemble that contains not only neural networks with 4 input variables, i.e., $X_{1}, X_{3}, X_{6}$, and $X_{8}$, but also networks with 3 input variables, i.e., $X_{1}, X_{3}$, and $X_{6}$ and $X_{1}, X_{6}$, and $X_{8}$.

Input selection can be viewed as a first step towards the bigger challenge of knowledge extraction, where one searches for rules describing the problem at hand [see for example (Setiono \& Liu 1997)]. So, although our input selection algorithm might be improved and generalised, we think that future research should investigate whether techniques, similar to those presented in this paper, can be used to obtain better and more stable rules by considering an ensemble instead of a single neural network.

\section{Acknowledgements}

We would like to thank Frans Boselie, Wim Wiegerinck and two anonymous referees for their useful comments on an earlier version of this paper.

\section{References}

Belsley, D. A., Kuh, E. \& Welsch, R. E. (1980). Regression diagnostics: identifying influential data and sources of collinearity, Wiley Series in Probability and Mathematical Statistics, Wiley, New York.

Belue, L. M. \& Bauer, Jr., K. W. (1995). Determining input features for multilayer perceptrons, Neurocomputing 7(2): 111-121.

Breiman, L. (1996). Bagging predictors, Machine Learning 24(2): 123-140.

Draper, N. R. \& Smith, H. (1981). Applied Regression Analysis, Wiley Series in Probability and Mathematical Statistics, second edn, Wiley, New York.

Friedman, J. H. (1991). Multivariate adaptive regression splines, The Annals of Statistics 19(1): 1-141.

Glorfeld, L. W. (1996). A methodology for simplification and interpretation of backpropagation-based neural network models, Expert Systems With Applications 10(1): 37-54. 
Grandvalet, Y. \& Canu, S. (1997). Adaptive noise injection for input variables relevance determination, Artificial Neural Networks - ICANN'97, Vol. 1327 of Lecture Notes in Computer Science, Springer, pp. 463-468.

Heskes, T. (1997). Balancing between bagging and bumping, in M. C. Mozer, M. I. Jordan \& T. Petsche (eds), Advances in Neural Information Processing Systems 9: Proceedings of the 1996 Conference, MIT Press, Cambridge, pp. $466-472$.

Hofmann, R. \& Tresp, V. (1996). Discovering structure in continuous variables using Bayesian networks, in D. S. Touretzky, M. C. Mozer \& M. E. Hasselmo (eds), Advances in Neural Information Processing Systems 8: Proceedings of the 1995 Conference, MIT Press, Cambridge, pp. 500-506.

Kleinbaum, D. G., Kupper, L. L. \& Muller, K. E. (1988). Applied Regression Analysis and Other Multivariable Methods, The Duxbury series in statistics and decision sciences, second edn, PWS-KENT Publishing Company, Boston.

Refenes, A.-P. N., Zapranis, A. D. \& Utans, J. (1996). Neural model identification, variable selection and model adequacy, in A. Weigend et al. (ed.), Neural Networks in Financial Engineering.

Setiono, R. \& Liu, H. (1997). Neurolinear: From neural networks to oblique decision rules, Neurocomputing 17(1): 1-24.

van de Laar, P., Gielen, S. \& Heskes, T. (1997). Input selection with partial retraining, Artificial Neural Networks - ICANN'97, Vol. 1327 of Lecture Notes in Computer Science, Springer, pp. 469-474.

Wolberg, W. H. \& Mangasarian, O. L. (1990). Multisurface method of pattern separation for medical diagnosis applied to breast cytology, Proceedings of the National Academy of Sciences USA 87: 9193-9196. 